\title{
Inter-domain Identity-based Proxy Re-encryption
}

\author{
Qiang Tang, Pieter Hartel, Willem Jonker \\ Faculty of EWI, University of Twente, the Netherlands \\ \{q.tang, pieter.hartel, jonker\}@utwente.nl
}

August 19, 2008

\begin{abstract}
Proxy re-encryption is a cryptographic primitive developed to delegate the decryption right from one party (the delegator) to another (the delegatee). So far, research efforts have only been devoted to the intra-domain setting, where the delegator and the delegatee are registered in the same domain. In this paper, we investigate the proxy re-encryption in the inter-domain setting, where the delegator and the delegatee are from different domains, and focus on the identity-based case. We analyze the trust relationships and possible threats to the plaintext privacy, and provide rigorous security definitions. We propose a new inter-domain identity-based proxy re-encryption scheme and prove its security in our security model. An interesting property of the proposed scheme is that, to achieve the chosen plaintext security for the delegator, the delegatee's IBE only needs to be one-way.
\end{abstract}

\section{Introduction}

Mambo and Okamoto [10] firstly propose the concept of delegation of decryption right in the context of speeding up decryption operations. Blaze, Bleumer and Strauss [2] introduce the concept of atomic proxy cryptography which is proxy reencryption. In a proxy re-encryption scheme, a delegator (say, Alice) and a delegatee (say, Bob) generate a proxy key that allows a semi-trusted third party (say, the proxy) to convert ciphertexts encrypted for Alice into ciphertexts which can be decrypted by Bob. Blaze, Bleumer and Strauss present a proxy re-encryption scheme based on Elgamal [6]. In their scheme, the proxy is also capable of converting ciphertexts encrypted for Bob into ciphertexts which can be decrypted by Alice. Jakobsson [9] and Zhou et al. [16] simultaneously propose quorum-based protocols, which divide the proxy into many components. Dodis and Ivan [8] propose generic constructions of proxy re-encryption schemes by using double-encryption. Ateniese et al. [1] propose an Elgamal-based scheme and show its application in securing file systems. In addition, Ateniese et al. also point out a number of desirable properties for proxy re-encryption schemes. Note that these papers mainly focus on the traditional public-key encryption schemes.

Since Shamir [12] firstly propose the concept, Identity-Based Encryption (IBE) has become a powerful tool in both theoretical cryptography and practical applications, especially after the work by Boneh and Franklin [4]. Considering their 
usefulness, it is interesting to extend the concept of proxy re-encryption into the identity-based setting, i.e. ID-based proxy re-encryption. Until now, apart from the generic construction of Dodis and Ivan [8], there are two identity-based proxy re-encryption schemes, in which the delegator and the delegatee are registered in the same domain. One is proposed by Green and Ateniese [7] and the other is proposed by Matsuo [11]. In both schemes, the delegator and the delegatee are assumed to be registered at the same domain (or, the same Key Generation Center (KGC)).

\subsection{Motivation and Contribution}

Proxy re-encryption has many promising applications including access control in file storage [1], email forwarding [15], and law enforcement [8]. For many cases of these applications, it would be more reasonable to assume an inter-domain setting where the delegator and the delegatee are from different domains than a intra-domain setting where all parties are from the same domain.

For example, Alice from university A (Alice's domain) might want to exploit an ID-based proxy re-encryption scheme so that messages encrypted under her identifier can be "automatically" converted into ciphertexts for her friend Bob from company $B$ (Bob's domain). In this example, it is unrealistic to assume that university $A$ and company $B$ share the same $K G C$.

In the inter-domain setting, existing ID-based proxy re-encryption schemes (e.g. those in $[7,11]$ ) cannot be used because the delegator and the delegatee are required to be registered at the same domain. To our knowledge, no particular research efforts have been devoted to proxy re-encryption in the inter-domain ID-based setting.

In this paper, we analyze the trust relationships and possible threats to the plaintexts of both the delegator and the delegatee for proxy re-encryption in the interdomain setting, and provide rigorous security definitions. Compared with the intradomain formulations [7, 11], our formulation of inter-domain proxy re-encryption has the following differences.

1. In our case, the delegator and the delegatee are from different domains, while they are assumed to be from the same domain in previous formulations in $[7,11]$.

2. In our model, the proxy key can be generated by either the delegator himself (the case in [7]) or the delegator together with the delegatee and even the KGCs. We believe this general assumption is more realistic in practice than that in [7].

3. As a result of the above assumption, the proxy key might leak some information about the delegatee's private key, hence, we have also taken into account the semantic security for the delegatee. This security formulation is necessary because the delegatee's IBE key might also be used for normal IBE services.

4. With respect to the definition of CPA security for the delegator, we have taken into account an ignored fact by previous works, i.e. a curious delegatee naturally has access to the plaintexts which have been re-encrypted by the proxy. 
We propose a new inter-domain identity-based proxy re-encryption scheme by extending the concept of the Green-Ateniese proxy re-encryption scheme IBP1 [7]. Given that the delegatee's IBE is IND-CPA secure, our scheme is secure against a chosen plaintext attack for the delegatee (IND-CPA secure). Given that the delegatee's IBE is one-way, we show that our scheme is secure against a chosen plaintext attack for the delegator (IND-CPA secure) based on the decision BDH assumption in the random oracle model. Interestingly, to achieve the chosen plaintext security for the delegator, the delegatee's IBE does not need to be IND-CPA secure.

\subsection{Organization}

The rest of the paper is organized as follows. In Section 2 we provide some preliminary knowledge on pairing and IBE. In Section 3 we present the security model for inter-domain ID-based proxy re-encryption. In Section 4 we present our new interdomain ID-based proxy re-encryption scheme and analyze its security. In Section 5 we conclude the paper.

\section{Preliminary}

We first review the necessary knowledge about pairing and the related assumptions. More detailed information can be found in the seminal paper [4]. A pairing (or, bilinear map) satisfies the following properties:

1. $\mathbb{G}$ and $\mathbb{G}_{1}$ are two multiplicative groups of prime order $p$;

2. $g$ is a generator of $\mathbb{G}$;

3. $\hat{e}: \mathbb{G} \times \mathbb{G} \rightarrow \mathbb{G}_{1}$ is an efficiently-computable bilinear map with the following properties:

- Bilinear: for all $u, v \in \mathbb{G}$ and $a, b \in \mathbb{Z}_{p}^{*}$, we have $\hat{e}\left(u^{a}, v^{b}\right)=\hat{e}(u, v)^{a b}$.

- Non-degenerate: $\hat{e}(g, g) \neq 1$.

As defined in [4], $\mathbb{G}$ is said to be a bilinear group if the group action in $\mathbb{G}$ can be computed efficiently and there exists a group $\mathbb{G}_{1}$ and an efficiently-computable bilinear map $\hat{e}$ as above.

The Bilinear Diffie-Hellman (BDH) problem in $\mathbb{G}$ is as follows: given a tuple $g, g^{a}, g^{b}, g^{c} \in \mathbb{G}$ as input, output $\hat{e}(g, g)^{a b c} \in \mathbb{G}_{1}$. An algorithm $\mathcal{A}$ has advantage $\epsilon$ in solving BDH in $\mathbb{G}$ if

$$
\operatorname{Pr}\left[\mathcal{A}\left(g, g^{a}, g^{b}, g^{c}\right)=\hat{e}(g, g)^{a b c}\right] \geq \epsilon .
$$

Similarly, we say that an algorithm $\mathcal{A}$ has advantage $\epsilon$ in solving the decision BDH problem in $\mathbb{G}$ if

$$
\left|\operatorname{Pr}\left[\mathcal{A}\left(g, g^{a}, g^{b}, g^{c}, \hat{e}(g, g)^{a b c}\right)=0\right]-\operatorname{Pr}\left[\mathcal{A}\left(g, g^{a}, g^{b}, g^{c}, T\right)=0\right]\right| \geq \epsilon .
$$

where the probability is over the random choice of $a, b, c \in \mathbb{Z}_{p}^{*}$, the random choice of $T \in \mathbb{G}_{1}$, and the random bits of $\mathcal{A}$. 
Definition 1. We say that the (decision) $(t, \epsilon)-B D H$ assumption holds in $\mathbb{G}$ if no t-time algorithm has advantage at least $\epsilon$ in solving the (decision) BDH problem in $\mathbb{G}$.

Given a security parameter $k$, a problem (say, BDH) is said to be intractable if any adversary has only negligible advantage in reasonable time. We usually define a scheme to be secure if any adversary has only a negligible advantage in the underlying security model. The time parameter is usually ignored.

Definition 2. The function $P(k): \mathbb{Z} \rightarrow \mathbb{R}$ is said to be negligible if, for every polynomial $f(k)$, there exists an integer $N_{f}$ such that $P(k) \leq \frac{1}{f(k)}$ for all $k \geq N_{f}$.

In IBE, we assume a Trusted Key Generation Center (KGC) will generate the public system parameter and dynamically issue private keys for users. An IBE scheme consists of four algorithms (Setup, Extract, Encrypt, Decrypt).

- $\operatorname{Setup}(k)$ : Run by the KGC, this algorithm takes a security parameter $k$ as input and generates the public parameter is params and a master key $m k$. The public parameter params is an implicit input for other algorithms and we omit it in the description for simplicity.

- Extract $(i d, m k)$ : Run by the KGC, this algorithm takes an identifier $i d$ and the master key $m k$ as input, and outputs the private key $s k_{i d}$ corresponding to $i d$.

- Encrypt $(m, i d)$ : Run by the message sender, this algorithm takes a message $m$ and an identifier $i d$ as input, and outputs a ciphertext $c$ encrypted under the public key corresponding to $i d$. Suppose that the plaintext space is $\mathcal{M}$.

- Decrypt $\left(c, s k_{i d}\right)$ : Run by the user with identifier $i d$, this algorithm takes a ciphertext $c$ and the private key $s k_{i d}$ as input, and outputs the message $m$.

The semantic security against an adaptive chosen plaintext attack (IND-CPA) is modelled by an IND-CPA game between a challenger and an adversary, where the challenger simulates the protocol execution and answers the queries from the adversary. Similarly, we can also define the one-wayness for IBE. Both attack games are depicted in Figure 1, and detailed explanations can be found in [4].

Note that, in both games, the adversary is not allowed to issue a query to the Extract oracle with the input $i d^{*}$. We assume the parameter params contains the state information generated during the experiment.

Definition 3. An IBE scheme is said to be semantically secure against an adaptive chosen plaintext attack (IND-CPA) if any polynomial time adversary's advantage is negligible in the IND-CPA game, where the advantage is defined to be $\left|\operatorname{Pr}\left[b^{\prime}=b\right]-\frac{1}{2}\right|$.

Definition 4. An IBE scheme is said to be one-way if any polynomial time adversary's advantage is negligible in the One-Wayness game, where the advantage is defined to be $\operatorname{Pr}\left[m^{\prime}=m\right]$. 

1. $($ params,$m k) \stackrel{\$}{\leftarrow} \operatorname{Setup}(k)$
1. $($ params,$m k) \stackrel{\$}{\leftarrow} \operatorname{Setup}(k)$
2. $\left(m_{0}, m_{1}, i d^{*}\right) \stackrel{\$}{\leftarrow} \mathcal{A}^{\text {(Extract) }}($ params $)$
2. $i d^{*} \stackrel{\$}{\longleftarrow} \mathcal{A}^{\text {(Extract) }}$ (params)
3. $b \stackrel{\$}{\leftarrow}\{0,1\} ; c^{*} \stackrel{\$}{\leftarrow} \operatorname{Encrypt}\left(m_{b}, i d^{*}\right)$
3. $m \stackrel{\$}{\leftarrow} \mathcal{M} ; c^{*} \stackrel{\$}{\leftarrow} \operatorname{Encrypt}\left(m, i d^{*}\right)$
4. $b^{\prime} \stackrel{\$}{\leftarrow} \mathcal{A}^{(\text {Extract })}\left(\right.$ params,$\left.c^{*}\right)$
4. $m^{\prime} \stackrel{\$}{\leftarrow} \mathcal{A}^{(\text {Extract })}\left(\right.$ params,$\left.c^{*}\right)$
IND-CPA
One-Wayness

Figure 1: Security Definitions for IBE

\section{Inter-domain ID-based Proxy Re-encryption}

Analogous to the traditional proxy re-encryption schemes (e.g. [1, 2]), an interdomain ID-based proxy re-encryption scheme allows a proxy to convert ciphertexts for an IBE user into ciphertexts for another IBE user, where the IBE users are from two different domains. In practice, there might be multiple different parties who play the role of proxy. For example, Alice may choose a party to delegate her decryption right to Bob and Eve may choose a different party to delegate his decryption right to Charlie, while these two proxy parties have no relationship. For the simplicity of description, we only assume one proxy in our security analysis and this proxy is given all the proxy keys.

Suppose that the delegator is registered at $\mathrm{KGC}_{1}$ with an IBE scheme

$$
\left(\text { Setup }_{1}, \text { Extract }_{1}, \text { Encrypt }_{1}, \text { Decrypt }_{1}\right)
$$

and the delegatee is registered at $\mathrm{KGC}_{2}$ with another IBE scheme

$$
\left(\text { Setup }_{2}, \text { Extract }_{2}, \text { Encrypt }_{2}, \text { Decrypt }_{2}\right) \text {. }
$$

As a result, there are five types of parties involved in the system: $\mathrm{KGC}_{1}$, the delegator (and IBE users in the delegator's domain), the proxy, $\mathrm{KGC}_{2}$, and the delegatee (and IBE users in the delegatee's domain). Apart from the IBE algorithms, an inter-domain IBE proxy re-encryption scheme consists of the following two new algorithms:

- Pextract $\left(i d, i d^{\prime}, s k_{i d},\left\{s k_{i d^{\prime}}, m k_{1}, m k_{2}\right\}\right)$ : This algorithm takes the delegator's identifier $i d$, the delegatee's identifier $i d$ ', the delegator's private key $s k_{i d}$, and possibly also $\left\{s k_{i d^{\prime}}, m k_{1}, m k_{2}\right\}$ as input and outputs the proxy key $r k_{i d \rightarrow i d^{\prime}}$ to the proxy. This algorithm will be run by the delegator and possibly with other parties, such as the delegatee and KGCs.

- Preenc $\left(c, r k_{i d \rightarrow i d^{\prime}}\right)$ : Run by the proxy, this algorithm takes a ciphertext $c$ for the delegator and the proxy key $r k_{i d \rightarrow i d^{\prime}}$ as input, and outputs a new ciphertext $c^{\prime}$ for the delegatee. 
Compared with that of Green and Ateniese [7], we have made the definition of Pextract a more general one. This definition has made the semantic security definition for the delegatee necessary, because the proxy key may leak some information on the delegatee's private key. The definition given by Matsuo [11] might be as general as ours, but the semantic security for the delegatee has been ignored. In the Appendix A, we show that a scheme, which has proven secure under the definition of Matsuo [11], may be insecure in practice.

\subsection{Threat Model}

We assume that both $\mathrm{KGC}_{1}$ and $\mathrm{KGC}_{2}$ are fully trusted. As mentioned in [5], the key escrow problem of IBE can be avoided by applying some standard techniques (such as secret sharing) to the underlying scheme, hence, we skip a formal discussion of this problem in this paper. We identify the following security requirements with respect to plaintext privacy.

1. The involved proxy is assumed to be curious in the following sense: it will honestly convert the delegator's ciphertexts using the proxy key; however, it might be curious to obtain some information about the plaintexts of the delegator and the delegatee. Ideally, the proxy should not obtain any information about the plaintexts of either the delegator or the delegatee.

2. The delegatee should be able to decrypt all the appropriate type of plaintexts of the delegator after the re-encryption by the proxy. However, the delegatee alone should not obtain any information about the plaintexts before the reencrypted by the proxy. This is essential when we want the proxy to be a policy enforcer.

3. Besides the re-encrypted ciphertexts from the delegator, a delegatee might also receive messages which are encrypted directly using his public key. The delegator and the proxy should not obtain any information about these messages.

In our formal definitions, the first and second requirements lead to the IND-CPA security for the delegator, and the third requirement leads to the IND-CPA security for the delegatee.

\subsection{Formal Semantic Security Definitions}

Semantic security for the delegator. In standard CPA security formulation for IBE (e.g. that in Section 2), the adversary is restricted from issuing any decryption query while it is allowed to obtain the ciphertext for any plaintext query (by running the encryption function). For the CPA security formulation for inter-domain proxy re-encryption, we want to apply the same restriction so that the adversary (either a curious proxy or a curious delegatee) is restricted from issuing any Decrypt ${ }_{1}$, Decrypt $_{2}$, and Preenc query. Note that, for a malicious delegatee, a Preenc query is equivalent to a Decrypt ${ }_{1}$ query. In our case, the adversary is allowed to issue Preenc ${ }^{\dagger}$ query to obtain the re-encrypted ciphertext for any plaintext. This oracle query models the situation that a curious delegatee naturally has access to the plaintexts 
which have been re-encrypted by the proxy. This issue has been ignored in the CPA security formulation in [7].

As a standard practice, the security is evaluated by an attack game played between a challenger and an adversary, where the challenger simulates the protocol execution and answers the queries from the adversary. Note that the allowed queries for the adversary reflects the adversary's capability in practice.

Definition 5. An inter-domain ID-based proxy re-encryption scheme is said to be IND-CPA secure if any polynomial time adversary has only a negligible advantage in the IND-CPA game, where the advantage is defined to be $\left|\operatorname{Pr}\left[b^{\prime}=b\right]-\frac{1}{2}\right|$.

1. $\left(\right.$ params $\left._{1}, m k_{1}\right) \stackrel{\$}{\longleftarrow} \operatorname{Setup}_{1}(k) ;\left(\right.$ params $\left._{2}, m k_{2}\right) \stackrel{\$}{\leftarrow} \operatorname{Setup}_{2}(k)$

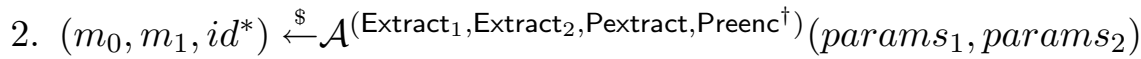

3. $b \stackrel{\$}{\leftarrow}\{0,1\} ; c^{*} \stackrel{\$}{\leftarrow} \operatorname{Encrypt}_{1}\left(m_{b}, i d^{*}\right)$

4. $b^{\prime} \stackrel{\$}{\leftarrow} \mathcal{A}^{\left(\text {Extract }_{1}, \text { Extract }_{2}, \text { Pextract, }, \text { Preenc }^{\dagger}\right)}\left(\right.$ params $_{1}$, params $\left._{2}, c^{*}\right)$

Figure 2: Semantic security for the delegator

As depicted in Figure 2, the IND-CPA game is as follows.

1. Game setup: The challenger takes a security parameter $k$ as input, runs the Setup $_{1}$ algorithm to generate the public system parameter params $s_{1}$ and the master key $m k_{1}$, and runs the Setup ${ }_{2}$ algorithm to generate the public system parameter params $_{2}$ and the master key $m k_{2}$.

2. Phase 1: The adversary takes params 1 and params $_{2}$ as input, and is allowed to issue the following types of oracle queries:

(a) Extract 1 query with any identifier $i d$ : The challenger returns the private key $s k_{i d}$ corresponding to $i d$.

(b) Extract 2 query with any identifier $i d^{\prime}$ : The challenger returns the private key $s k_{i d^{\prime}}$ corresponding to $i d^{\prime}$.

(c) Pextract query with $\left(i d, i d^{\prime}\right)$ : The challenger returns the delegation key $r k_{i d \rightarrow i d^{\prime}}$.

(d) Preenc ${ }^{\dagger}$ query with $\left(m, i d, i d^{\prime}\right)$ : The challenger computes $c=\operatorname{Encrypt}_{1}(m, i d)$ and then returns a new ciphertext $c^{\prime}=\operatorname{Preenc}\left(c, r k_{i d \rightarrow i d^{\prime}}\right)$.

Once the adversary decides that Phase 1 is over, it outputs two equal length plaintexts $m_{0}, m_{1}$ and an identifier $i d^{*}$ on which it wishes to be challenged. There are two constraints here. The first one is that $i d^{*}$ has not been the input to any Extract 1 query. The second one is that, at the end of Phase 1, for any $i d^{\prime}$, if $\left(i d^{*}, i d^{\prime}\right)$ has been the input to a Pextract query then $i d^{\prime}$ should not have been the input to any Extract 2 query. 
3. Challenge: The challenger picks a random bit $b \in\{0,1\}$ and return $c^{*}=$ Encrypt $_{1}\left(m_{b}, i d^{*}\right)$ as the challenge to the adversary.

4. Phase 2: The adversary is allowed to continue issuing the same types of queries as in Phase 1 but with the following constraints. The adversary is not allowed to issue any Extract ${ }_{1}$ query with $i d^{*}$. At the end of Phase 2, for any $i d^{\prime}$, if $\left(i d^{*}, i d^{\prime}\right)$ has been the input to a Pextract query then $i d^{\prime}$ should not be the input to any Extract 2 query.

5. Guess (game ending): The adversary outputs a guess $b^{\prime} \in\{0,1\}$.

In this attack game for CPA security, the adversary (either a curious proxy or a curious delegatee) has been given the maximum privilege under the condition that it should not trivially win the game. If the adversary acts as a malicious proxy, the adversary is allowed to obtain any proxy keys and IBE keys from both domains, except for the trivial cases: obtain the private key $s k_{i d^{*}}$ from $\mathrm{KGC}_{1}$ 's domain or a delegatee's private key for which the adversary knows the proxy key. If the adversary acts as a malicious delegatee, the adversary is allowed to obtain any proxy keys and IBE keys from both domain and access to the Preenc ${ }^{\dagger}$ oracle, except for the trivial cases: obtain the private key $s k_{i d^{*}}$ from $\mathrm{KGC}_{1}$ 's domain or the proxy key for which the adversary knows the IBE private key.

Semantic security for the delegatee. Similarly, we can define the chosen plaintext security for the delegatee and the corresponding IND-CPA game is depicted in Figure 3.

Definition 6. An inter-domain ID-based proxy re-encryption scheme is said to be IND-CPA secure if any polynomial time adversary has only a negligible advantage in the IND-CPA game, where the advantage is defined to be $\left|\operatorname{Pr}\left[b^{\prime}=b\right]-\frac{1}{2}\right|$.

1. $\left(\right.$ params $\left._{1}, m k_{1}\right) \stackrel{\$}{\leftarrow} \operatorname{Setup}_{1}(k) ;\left(\right.$ params $\left._{2}, m k_{2}\right) \stackrel{\$}{\leftarrow} \operatorname{Setup}_{2}(k)$

2. $\left(m_{0}, m_{1}, i d^{*}\right) \stackrel{\$}{\leftarrow} \mathcal{A}^{\left(\text {Extract }_{1}, \text { Extract }_{2}, \text { Pextract }\right)}\left(\right.$ params $_{1}$, params $\left._{2}\right)$

3. $b \stackrel{\$}{\leftarrow}\{0,1\} ; c^{*} \stackrel{\$}{\leftarrow} \operatorname{Encrypt}_{2}\left(m_{b}, i d^{*}\right)$

4. $b^{\prime} \stackrel{\$}{\leftarrow} \mathcal{A}^{\left(\text {Extract }_{1}, \text { Extract }_{2}, \text { Pextract }\right)}\left(\right.$ params $_{1}$, params $\left._{2}, c^{*}\right)$

Figure 3: Semantic security for the delegatee

Note that, in this attack game, the adversary is not allowed to issue a query to

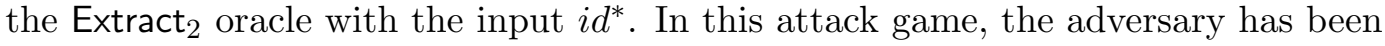
given the maximum privilege under the condition that it should not trivially win the game because the adversary is allowed to obtain any proxy keys and IBE keys from both domain, except for the trivial cases: obtain the private key $s k_{i d^{*}}$ from $\mathrm{KGC}_{2}$ 's domain. 
According to our definition, if an inter-domain ID-based proxy re-encryption scheme achieves IND-CPA security for the delegatee, then it is uni-directional according to the definition in [1], i.e. delegation from the delegator to the delegatee does not allow re-encryption (using the same proxy key) from the delegatee to the delegator.

\section{An inter-domain ID-based proxy re-encryption scheme}

In this section we propose a new inter-domain ID-based proxy re-encryption scheme by extending the concept of the Green-Ateniese proxy re-encryption scheme IBP1 [7]. We then prove its security in our security model.

\subsection{Description of our scheme}

The delegator uses a variant of the Boneh-Franklin IBE scheme [4]. Similar modifications are also made in [7] and they are essential for us to construct proxy reencryption schemes.

1. Setup ${ }_{1}(k)$ : Run by the KGC, this algorithm takes a security parameter $k$ as input and generates two cyclic groups $\mathbb{G}$ and $\mathbb{G}_{1}$ of prime order $p$, a generator $g$ of $\mathbb{G}$, a bilinear map $\hat{e}: \mathbb{G} \times \mathbb{G} \rightarrow \mathbb{G}_{1}$, a master secret key $\alpha \in \mathbb{Z}_{p}^{*}$, and a hash function $\mathrm{H}_{1}:\{0,1\}^{*} \rightarrow \mathbb{G}$. The public parameter is params $=\left(\mathbb{G}, \mathbb{G}_{1}, p, g, \mathrm{H}_{1}, \hat{e}, p k\right)$, where $p k=g^{\alpha}$ is the public key of the KGC.

In the original Boneh-Franklin scheme, the plaintext space is $\{0,1\}^{n}$ where $n$ is an integer and there is an additional hash function $\mathrm{H}_{2}: \mathbb{G}_{1} \rightarrow\{0,1\}^{n}$.

2. Extract $1(i d)$ : Run by the KGC, this algorithm takes an identifier $i d \in\{0,1\}^{*}$ and the master key $m k$ as input, and outputs the private key $s k_{i d}=p k_{i d}^{\alpha}$, where $p k_{i d}=\mathrm{H}_{1}(i d)$.

3. $\operatorname{Encrypt}_{1}(m, i d)$ : Run by the message sender, this algorithm takes a message $m \in \mathbb{G}_{1}$ and an identifier $i d \in\{0,1\}^{*}$ as input, and outputs the ciphertext $c=\left(c_{1}, c_{2}\right)$ where $r \in \mathbb{Z}_{p}^{*}, c_{1}=g^{r}$, and $c_{2}=m \cdot \hat{e}\left(p k_{i d}, p k\right)^{r}$.

In the original Boneh-Franklin scheme, $c_{2}=m \oplus \mathrm{H}_{2}\left(\hat{e}\left(p k_{i d}, p k\right)^{r}\right)$.

4. $\operatorname{Decrypt}_{1}\left(c, s k_{i d}\right)$ : Run by the receiver with identifier $i d$, this algorithm takes a ciphertext $c=\left(c_{1}, c_{2}\right)$ and the private key $s k_{i d}$ as input, and outputs the message $m=\frac{c_{2}}{\hat{e}\left(s k_{i d}, c_{1}\right)}$.

In the original Boneh-Franklin scheme, $m=c_{2} \oplus \mathrm{H}_{2}\left(\hat{e}\left(s k_{i d}, c_{1}\right)\right)$.

Suppose that the delegator is registered at $\mathrm{KGC}_{1}$ with the above IBE scheme, and possesses identifier and private key pair $\left(i d, s k_{i d}\right) . \mathrm{KGC}_{1}$ publishes another hash function $\mathrm{H}_{2}:\{0,1\}^{*} \rightarrow \mathbb{G}$. Suppose that the delegatee is registered at $\mathrm{KGC}_{2}$ with another IBE scheme $\left(\right.$ Setup $_{2}$, Extract ${ }_{2}$, Encrypt $_{2}$, Decrypt $\left._{2}\right)$, and possesses identifier and private key pair $\left(i d^{\prime}, s k_{i d^{\prime}}\right)$. Suppose also that this IBE scheme has message space $\mathcal{M}_{2}$. 
If the delegator wants to delegate his decryption right to the delegatee, the algorithms are as follows.

- Pextract $\left(i d, i d^{\prime}, s k_{i d}\right)$ : Run by the delegator, this algorithm outputs the proxy key $r k_{i d \rightarrow i d^{\prime}}=\left(s k_{i d}^{-1} \cdot \mathrm{H}_{2}(X), e_{i d \rightarrow i d^{\prime}}\right)$, where $X \in_{R} \mathcal{M}_{2}$ and $e_{i d \rightarrow i d^{\prime}}=\operatorname{Encrypt}_{2}\left(X, i d^{\prime}\right)$.

- Preenc $\left(c, r k_{i d \rightarrow i d^{\prime}}\right):$ Run by the proxy, this algorithm takes a ciphertext $c=$ $\left(c_{1}, c_{2}\right)$, where $c_{1}=g^{r}$ and $c_{2}=m \cdot \hat{e}\left(p k_{i d}, p k\right)^{r}$, and $r k_{i d \rightarrow i d^{\prime}}=\left(s k_{i d}^{-1}\right.$. $\left.\mathrm{H}_{2}(X), e_{i d \rightarrow i d^{\prime}}\right)$ as input, and outputs a new ciphertext $c^{\prime}=\left(c_{1}^{\prime}, c_{2}^{\prime}, c_{3}^{\prime}\right)$ for the delegatee, where

$$
\begin{aligned}
& c_{1}^{\prime}=\operatorname{Encrypt}_{2}\left(c_{1}, i d^{\prime}\right), c_{3}^{\prime}=e_{i d \rightarrow i d^{\prime}}, \\
c_{2}^{\prime}= & c_{2} \cdot \hat{e}\left(c_{1}, s k_{i d}^{-1} \cdot \mathrm{H}_{2}(X) \cdot \mathrm{H}_{2}\left(c_{1}\right)\right) \\
= & m \cdot \hat{e}\left(p k_{i d}, p k\right)^{r} \cdot \hat{e}\left(c_{1}, s k_{i d}^{-1} \cdot \mathrm{H}_{2}(X) \cdot \mathrm{H}_{2}\left(c_{1}\right)\right) \\
= & m \cdot \hat{e}\left(c_{1}, \mathrm{H}_{2}(X) \cdot \mathrm{H}_{2}\left(c_{1}\right)\right) .
\end{aligned}
$$

Given a re-encrypted ciphertext $c^{\prime}$, the delegatee can obtain the plaintext $m$ by computing:

$$
\begin{aligned}
m^{\prime} & =\frac{c_{2}^{\prime}}{\hat{e}\left(\operatorname{Decrypt}_{2}\left(c_{1}^{\prime}, s k_{i d^{\prime}}\right), \mathrm{H}_{2}\left(\operatorname{Decrypt}_{2}\left(c_{3}^{\prime}, s k_{i d^{\prime}}\right)\right) \cdot \mathrm{H}_{2}\left(\operatorname{Decrypt}_{2}\left(c_{1}^{\prime}, s k_{i d^{\prime}}\right)\right)\right)} \\
& =\frac{m \cdot \hat{e}\left(c_{1}, \mathrm{H}_{2}(X) \cdot \mathrm{H}_{2}\left(c_{1}\right)\right)}{\hat{e}\left(c_{1}, \mathrm{H}_{2}(X) \cdot \mathrm{H}_{2}\left(c_{1}\right)\right)} \\
& =m .
\end{aligned}
$$

Note that, to decrypt a re-encrypted ciphertext $c^{\prime}$, the delegatee needs to obtain in advance $\mathrm{KGC}_{1}$ 's public parameter $\left(\mathbb{G}, \mathbb{G}_{1}, g, p, \hat{e}, \mathrm{H}_{1}, \mathrm{H}_{2}\right)$.

The proposed inter-domain ID-based proxy re-encryption scheme differs from the Green-Ateniese proxy re-encryption scheme IBP1 [7] in the following aspects:

1. In our scheme, the delegator and the delegatee are from two different domains, while they are required to be the same domain in the IBP1.

2. In the Green-Ateniese proxy re-encryption scheme IBP1, the algorithm Preenc outputs $c_{1}^{\prime}=c_{1}$ and $c_{2}^{\prime}=c_{2} \cdot \hat{e}\left(c_{1}, s k_{i d}^{-1} \cdot \mathrm{H}_{2}(X)\right)$. Our modifications in the above scheme are essential for us to prove the IND-CPA security. Note that under our security definition the adversary is allowed access to the Preenc ${ }^{\dagger}$ oracle, which is different from that in [7]. Without the modifications, we cannot prove our result.

\subsection{Analysis of the general construction}

Since the delegator generates the proxy key on his own, therefore, from Definition 3 and Definition 6, the following result is straightforward.

Lemma 1. Given that $\left(\right.$ Setup $_{2}$, Extract ${ }_{2}$, Encrypt ${ }_{2}$, Decrypt $\left.{ }_{2}\right)$ is IND-CPA secure, the proposed inter-domain ID-based proxy re-encryption scheme is IND-CPA secure. 
Lemma 2. Given that $\left(\right.$ Setup $_{2}$, Extract ${ }_{2}$, Encrypt $_{2}$, Decrypt $\left._{2}\right)$ is one-way, the proposed inter-domain ID-based proxy re-encryption scheme is IND-CPA secure for the delegator based on the decision BDH assumption in the random oracle model.

Proof sketch. We suppose that the total number of queries issued to $\mathrm{H}_{1}$ and $\mathrm{H}_{2}$ is bounded by integer $q_{1}$ and $q_{2}$, respectively ${ }^{1}$. Suppose an adversary $\mathcal{A}$ acting as a malicious delegatee has the non-negligible advantage $\epsilon$ in the IND-CPA game. The security proof is done through a sequence of games [13].

Game $_{0}$ : In this game, the challenger faithfully simulates the protocol execution and answers the oracle queries from $\mathcal{A}$. the challenger simulates the random oracle $\mathrm{H}_{1}$ as follows: the challenger maintains a list of vectors, each of them containing a request message, an element of $\mathbb{G}$ (the hash-code for this message), and an element of $\mathbb{Z}_{p}^{*}$. After receiving a request message, the challenger first checks its list to see whether the request message is already in the list. If the check succeeds, the challenger returns the stored element of $\mathbb{G}$; otherwise, the challenger returns $g^{y}$, where $y$ a randomly chosen element of $\mathbb{Z}_{p}^{*}$, and stores the new vector in the list. the challenger simulates the random oracle $\mathrm{H}_{2}$ as follows: the challenger maintains a list of vectors, each of them containing a request message and an element of $\mathbb{G}$ (the hash-code for this message). After receiving a request message, the challenger first checks its list to see whether the request message is already in the list. If the check succeeds, the challenger returns the stored element of $\mathbb{G}$; otherwise, the challenger returns $u$ which is a randomly chosen element of $\mathbb{G}$, and stores the new vector in the list.

Let $\delta_{0}=\operatorname{Pr}\left[b^{\prime}=b\right]$, as we assumed at the beginning, $\left|\delta_{0}-\frac{1}{2}\right|=\epsilon$.

Game $_{1}$ : In this game, the challenger performs as follows.

1. Game setup: the challenger faithfully simulates the setup phase.

2. Phase 1: the challenger randomly selects $j \in\left\{1,2, \cdots, q_{1}+1\right\}$. If $j=q_{1}+1$, the challenger faithfully answers the oracle queries from $\mathcal{A}$. If $1 \leq j \leq q_{1}$, we assume the $j$-th input to $\mathrm{H}_{1}$ is $\tilde{i d}$ and the challenger answers the oracle queries from $\mathcal{A}$ as follows: Answer Extract ${ }_{1}$, Extract 2 , Pextract, and Preenc ${ }^{\dagger}$ faithfully, except that the challenger aborts as a failure when $\tilde{i d}$ is the input to a Extract query.

3. Challenge: After receiving $\left(m_{0}, m_{1}, i d^{*}\right)$ from the adversary, if one of the following events occurs, the challenger aborts as a failure.

(a) $i d^{*}$ has been issued to $\mathrm{H}_{1}$ as the $i$-th query and $i \neq j$,

(b) $i d^{*}$ has not been issued to $\mathrm{H}_{1}$ and $1 \leq j \leq q_{1}$.

Note that, if the adversary does not abort then either $1 \leq j \leq q_{1}$ and $i d^{*}=\tilde{i d}$ is the input to $j$-th $\mathrm{H}_{1}$ query or $j=q_{1}+1$ and $i d^{*}$ has not been the input to any $\mathrm{H}_{1}$ query. the challenger faithfully returns the challenge.

4. Phase 2: the challenger answers the oracle queries faithfully.

\footnotetext{
${ }^{1}$ For simplicity of description, it is reasonable to assume that the total number is counted for queries with different inputs.
} 
5. Guess (game ending): the adversary outputs a guess $b^{\prime} \in\{0,1\}$.

The probability that the challenger successfully ends is $\frac{1}{q_{1}+1}$, i.e. the probability that the challenger does not abort in its execution is $\frac{1}{q_{1}+1}$. Let $\delta_{1}=\operatorname{Pr}\left[b^{\prime}=b\right]$ when the challenger successfully ends, in which case $\left|\delta_{1}=\delta_{0}\right|$. Let $\theta_{1}$ be the probability that the challenger successfully ends and $b^{\prime}=b$. We have $\theta_{1}=\frac{\delta_{1}}{q_{1}+1}$.

Game $_{2}$ : In this game, the challenger simulates the protocol execution and answers the oracle queries from $\mathcal{A}$ in the following way.

1. Game setup: the challenger faithfully simulates the setup phase.

2. Phase 1: the challenger randomly selects $j \in\left\{1,2, \cdots, q_{1}+1\right\}$. If $j=q_{1}+1$, the challenger faithfully answers the oracle queries from $\mathcal{A}$. If $1 \leq j \leq q_{1}$, the challenger answers $j$-th query to $\mathrm{H}_{1}$ with $g^{\beta}$ where $\beta \in \in_{R} \mathbb{Z}_{p}^{*}$, and answers the oracle queries from $\mathcal{A}$ as follows. Suppose the input of the $j$-th query to $\mathrm{H}_{1}$ is $\tilde{i d}$. the challenger answers queries to Extract 1 , Extract 2 , Pextract, and Preenc ${ }^{\dagger}$ in the same way as in $\mathrm{Game}_{1}$, except for the following. the challenger keeps a list of vector $\left(i d^{\prime}, r k_{i d \rightarrow i d^{\prime}}, X_{i d^{\prime}}, X_{i d^{\prime}}^{\prime}, g_{i d^{\prime}}, h_{i d^{\prime}}\right)$.

(a) Pextract query with the input $\left(\tilde{i d}, i d^{\prime}\right)$ : If $r k_{\tilde{i d} \rightarrow i d^{\prime}}$ exists in one of the lists, the challenger returns the value. Otherwise, the challenger returns the proxy key $r k_{\tilde{i d} \rightarrow i d^{\prime}}$, where

$$
\begin{gathered}
X_{i d^{\prime}}, X_{i d^{\prime}}^{\prime} \in_{R} \mathcal{M}_{2}, g_{i d^{\prime}} \in_{R} \mathbb{G}, e_{\tilde{i d} \rightarrow i d^{\prime}}=\operatorname{Encrypt}_{2}\left(X_{i d^{\prime}}^{\prime}, i d^{\prime}\right), \\
\mathrm{H}_{2}\left(X_{i d^{\prime}}\right)=g_{i d^{\prime}}, r k_{\tilde{i d} \rightarrow i d^{\prime}}=\left(s k_{\tilde{i d}}^{-1} \cdot g_{i d^{\prime}}, e_{\tilde{i d} \rightarrow i d^{\prime}}\right) .
\end{gathered}
$$

the challenger adds the vector $\left(i d^{\prime}, r k_{\tilde{i d} \rightarrow i d^{\prime}}, X_{i d^{\prime}}, X_{i d^{\prime}}^{\prime}, g_{i d^{\prime}}, h_{i d^{\prime}}\right)$ to the list, where $h_{i d^{\prime}}$ is set to be a special symbol $\perp$.

(b) Preenc $c^{\dagger}$ query with the input $\left(m, \tilde{i d}, i d^{\prime}\right)$ : the challenger performs according to the following rules.

- If $r k_{\tilde{i d} \rightarrow i d^{\prime}}$ does not exist, the challenger generates $\left(i d^{\prime}, r k_{\tilde{i d} \rightarrow i d^{\prime}}, X_{i d^{\prime}}, X_{i d^{\prime}}^{\prime}, g_{i d^{\prime}}, h_{i d^{\prime}}\right)$, where

$$
\begin{gathered}
X_{i d^{\prime}}, X_{i d^{\prime}}^{\prime} \in_{R} \mathcal{M}_{2}, g_{i d^{\prime}} \in R \mathbb{G}, e_{\tilde{i d} \rightarrow i d^{\prime}}=\operatorname{Encrypt}_{2}\left(X_{i d^{\prime}}^{\prime}, i d^{\prime}\right), \\
\mathrm{H}_{2}\left(X_{i d^{\prime}}\right)=g_{i d^{\prime}}, r k_{\tilde{i d} \rightarrow i d^{\prime}}=\left(s k_{\tilde{i d}}^{-1} \cdot g_{i d^{\prime}}, e_{\tilde{i d} \rightarrow i d^{\prime}}\right) .
\end{gathered}
$$

the challenger then returns a new ciphertext $c^{\prime}=\left(c_{1}^{\prime}, c_{2}^{\prime}, c_{3}^{\prime}\right)$, where

$$
\begin{gathered}
t_{1}, c_{1}, g_{c_{1}} \in_{R} \mathbb{G}, \quad c_{1}^{\prime}=\operatorname{Encrypt}_{2}\left(c_{1}, i d^{\prime}\right), \\
c_{2}^{\prime}=m \cdot \hat{e}\left(t_{1}, g\right) \cdot \hat{e}\left(c_{1}, g_{c_{1}}\right), \quad c_{3}^{\prime}=e_{\tilde{i d} \rightarrow i d^{\prime}}, \quad h_{i d^{\prime}}=g_{i d^{\prime}} \cdot c_{1}^{-1} \cdot t_{1},
\end{gathered}
$$

and adds the new vector to the list.

- If $r k_{\tilde{i d} \rightarrow i d^{\prime}}$ exists in the list but $h_{i d^{\prime}}=\perp$ (which means a Pextract query with the input $\left(\tilde{i d}, i d^{\prime}\right)$ has been issued), the challenger then returns a new ciphertext $c^{\prime}=\left(c_{1}^{\prime}, c_{2}^{\prime}, c_{3}^{\prime}\right)$ faithfully. 
- If $r k_{\tilde{i d} \rightarrow i d^{\prime}}$ exists in the list and $h_{i d^{\prime}} \neq \perp$, the challenger then returns a new ciphertext $c^{\prime}=\left(c_{1}^{\prime}, c_{2}^{\prime}, c_{3}^{\prime}\right)$, where

$$
\begin{gathered}
c_{1}, g_{c_{1}} \in R \mathbb{G}, c_{1}^{\prime}=\operatorname{Encrypt}_{2}\left(c_{1}, i d^{\prime}\right), \\
c_{2}^{\prime}=m \cdot \hat{e}\left(c_{1} \cdot h_{i d^{\prime}}\right) \cdot \hat{e}\left(c_{1}, g_{c_{1}}\right), \quad c_{3}^{\prime}=e_{\tilde{i d} \rightarrow i d^{\prime}} .
\end{gathered}
$$

After a Preenc ${ }^{\dagger}$ query with the input $\left(m, \tilde{i d}, i d^{\prime}\right)$ and a Extract 2 query with the input $i d^{\prime}$ have been issued, the challenger returns $h_{i d^{\prime}}$ if $X_{i d^{\prime}}^{\prime}$ is issued to $\mathrm{H}_{2}$ and returns $g_{c_{1}}$ if $c_{1}$ is issued to $\mathrm{H}_{2}$.

3. Challenge: the challenger performs in the same way as in Game ${ }_{1}$, except that answers Pextract query with the input $\left(i d^{*}, i d^{\prime}\right)$ and Preenc ${ }^{\dagger}$ query with the input $\left(m, i d^{*}, i d^{\prime}\right)$ as in Phase 1.

4. Phase 2: the challenger answers the oracle queries from $\mathcal{A}$ as in Phase 1.

5. Guess (game ending): the adversary outputs a guess $b^{\prime} \in\{0,1\}$.

Let $\theta_{2}$ be the probability that the challenger successfully ends and $b^{\prime}=b$. Let $E_{1}$ be the event that, for some $i d^{\prime}$ and $m$, the adversary issues a $\mathrm{H}_{2}$ query with the input $X_{i d^{\prime}}^{\prime}$ query but there is no Extract 2 query with the input $i d^{\prime}$, or the adversary issues a $\mathrm{H}_{2}$ query with the input $X_{i d^{\prime}}^{\prime}$ query before any Preenc ${ }^{\dagger}$ query with the input $\left(m, \tilde{i d}, i d^{\prime}\right)$, or the adversary issues a $\mathrm{H}_{2}$ query with the input $c_{1}$ query but there is no Extract 2 query with the input $i d^{\prime}$. Compared with Game ${ }_{1}, G_{a m e}$ differs when $E_{1}$ occurs. From the difference lemma [13], we have $\left|\theta_{2}-\theta_{1}\right| \leq \epsilon_{2}=\operatorname{Pr}\left[E_{1}\right]$ which is negligible based on the one-wayness of $\left(\right.$ Setup $_{2}$, Extract $_{2}$, Encrypt $_{2}$, Decrypt $\left._{2}\right)$ in the random oracle model.

Game 3 : In this game, the challenger simulates the protocol execution and answers the oracle queries from $\mathcal{A}$ in the same way as in Game 2 , except for the following. For a Pextract query with the input $\left(i d^{*}, i d^{\prime}\right)$, the challenger returns the proxy key $r k_{i d^{*} \rightarrow i d^{\prime}}$, where $T_{i d^{\prime}}, X_{i d^{\prime}}^{\prime} \in R \mathbb{G}_{1}$ and

$$
r k_{i d^{*} \rightarrow i d^{\prime}}=\left(T_{i d^{\prime}}, \operatorname{Encrypt}_{2}\left(X_{i d^{\prime}}^{\prime}, i d^{\prime}\right)\right) .
$$

Let $\theta_{3}$ be the probability that the challenger successfully ends and $b^{\prime}=b$. Compared with Game 2 , we only change the notation of random value $s k_{\tilde{i d}}^{-1} \cdot g_{i d^{\prime}}$ with $T_{i d^{\prime}}$. As a result, we have $\left|\theta_{3}-\theta_{2}\right|=\epsilon_{3}=0$.

$\mathrm{Game}_{4}$ : In this game, the challenger simulates the protocol execution and answers the oracle queries from $\mathcal{A}$ in the same way as in $\mathrm{Game}_{3}$, except for the following. In the challenge phase the challenger returns $c^{*}=\left(c_{1}^{*}, c_{2}^{*}\right)$ as the challenge, where

$$
b \in_{R}\{0,1\}, r \in_{R} \mathbb{Z}_{p}^{*}, T \in_{R} \mathbb{G}_{1}, c_{1}^{*}=g^{r}, c_{2}^{*}=m_{b} \cdot T .
$$

Let $\theta_{4}$ be the probability that the challenger successfully ends and $b^{\prime}=b$. We have $\theta_{4}=\frac{1}{2\left(q_{1}+1\right)}$ since $T \in \in_{R} \mathbb{G}_{1}$. Compared with Game 3 , the only difference in Game $_{4}$ is that $\hat{e}(g, g)^{\alpha \cdot \beta \cdot r}$ is replaced with $T \in_{R} \mathbb{G}_{1}$ in the challenge phase. Using the interpolation method [13], we have $\left|\theta_{4}-\theta_{3}\right| \leq \epsilon_{4}$ which is negligible based on the decision $\mathrm{BDH}$ assumption. 
From $\left|\theta_{2}-\theta_{1}\right| \leq \epsilon_{2},\left|\theta_{3}-\theta_{2}\right| \leq \epsilon_{3},\left|\theta_{4}-\theta_{3}\right| \leq \epsilon_{4}$, and $\theta_{4}=\frac{1}{2\left(q_{1}+1\right)}$, we have $\left|\frac{1}{2\left(q_{1}+1\right)}-\theta_{1}\right| \leq \epsilon_{2}+\epsilon_{3}+\epsilon_{4}$. In addition, from $\left|\delta_{0}-\frac{1}{2}\right|=\epsilon,\left|\delta_{1}-\delta_{0}\right| \leq \epsilon_{1}$ and

$\theta_{1}=\frac{\delta_{1}}{q_{1}+1}$, we have $\frac{\epsilon}{q_{1}+1} \leq \frac{\epsilon_{1}}{q_{1}+1}+\epsilon_{2}+\epsilon_{3}+\epsilon_{4}$. Because $\epsilon_{i}(1 \leq i \leq 4)$ are negligible and $\epsilon$ is assumed to be non-negligible, we get a contradiction. As a result, the proposed inter-domain ID-based proxy re-encryption scheme is IND-CPA secure based on the decision $\mathrm{BDH}$ assumption in the random oracle model, given that $\left(\right.$ Setup $_{2}$, Extract $_{2}$, Encrypt $_{2}$, Decrypt $\left._{2}\right)$ is one-way.

\section{Conclusion}

In this paper, we have examined the concept of inter-domain ID-based proxy reencryption and proposed a chosen plaintext security security definitions. We have also proposed an inter-domain ID-based proxy re-encryption scheme which has the interesting property that, to achieve the chosen plaintext security for the delegator, the delegatee's IBE only needs to be one-way. In our security formulation, only chosen plaintext security has been defined, however this definition can be extended to model chosen ciphertext security by appropriately allowing Decrypt ${ }_{1}$, Decrypt $_{2}$, and Preenc queries to the adversary. It is an interesting future work to construct inter-domain ID-based proxy re-encryption schemes with chosen ciphertext security. It is also interesting to further investigate the application of inter-domain proxy re-encryption in emerging fields such as Personal Health Record (PHR) protection [14].

\section{References}

[1] G. Ateniese, K. Fu, M. Green, and S. Hohenberger. Improved proxy reencryption schemes with applications to secure distributed storage. ACM Trans. Inf. Syst. Secur., 9(1):1-30, 2006.

[2] M. Blaze, G. Bleumer, and M. Strauss. Divertible protocols and atomic proxy cryptography. In K. Nyberg, editor, Advances in Cryptology - EUROCRYPT '98, International Conference on the Theory and Application of Cryptographic Techniques, volume 1403 of Lecture Notes in Computer Science, pages 127-144. Springer, 1998.

[3] D. Boneh and X. Boyen. Efficient selective-id secure identity-based encryption without random oracles. In C. Cachin and J. Camenisch, editors, Advances in Cryptology - EUROCRYPT 2004, International Conference on the Theory and Applications of Cryptographic Techniques, volume 3027 of Lecture Notes in Computer Science, pages 223-238. Springer, 2004.

[4] D. Boneh and M. K. Franklin. Identity-based encryption from the weil pairing. In J. Kilian, editor, Advances in Cryptology - CRYPTO 2001, 21st Annual International Cryptology Conference, volume 2139 of Lecture Notes in Computer Science, pages 213-229. Springer, 2001. 
[5] L. Chen. An interpretation of identity-based cryptography. In A. Aldini and R. Gorrieri, editors, Foundations of Security Analysis and Design IV, FOSAD 2006/2007 Tutorial Lectures, volume 4677 of Lecture Notes in Computer Science, pages 183-208. Springer, 2007.

[6] T. ElGamal. A public key cryptosystem and a signature scheme based on discrete logarithms. In G. R. Blakley and D. Chaum, editors, Advances in Cryptology, Proceedings of CRYPTO '84, volume 196 of Lecture Notes in Computer Science, pages 10-18. Springer, 1985.

[7] M. Green and G. Ateniese. Identity-based proxy re-encryption. In J. Katz and M. Yung, editors, Applied Cryptography and Network Security, 5th International Conference, volume 4521 of Lecture Notes in Computer Science, pages 288-306. Springer, 2007.

[8] A. Ivan and Y. Dodis. Proxy cryptography revisited. In Proceedings of the Network and Distributed System Security Symposium. The Internet Society, 2003.

[9] Markus Jakobsson. On quorum controlled asymmetric proxy re-encryption. In H. Imai and Y. Zheng, editors, Public Key Cryptography, Second International Workshop on Practice and Theory in Public Key Cryptography, volume 1560 of Lecture Notes in Computer Science, pages 112-121. Springer, 1999.

[10] M. Mambo and E. Okamoto. Proxy cryptosystems: Delegation of the power to decrypt ciphertexts. IEICE TRANSACTIONS on Fundamentals of Electronics, Communications and Computer Sciences, E80-A(1):54-63, 1997.

[11] T. Matsuo. Proxy re-encryption systems for identity-based encryption. In T. Takagi, T. Okamoto, E. Okamoto, and T. Okamoto, editors, Pairing-Based Cryptography - Pairing 2007, First International Conference, volume 4575 of Lecture Notes in Computer Science, pages 247-267. Springer, 2007.

[12] A. Shamir. Identity-based cryptosystems and signature schemes. In Advances in Cryptology, Proceedings of CRYPTO '84, volume 196 of Lecture Notes in Computer Science, pages 47-53. Springer, 1985.

[13] V. Shoup. Sequences of games: a tool for taming complexity in security proofs. http://shoup.net/papers/, 2006.

[14] The US Department of Health and Human Services. Summary of the HIPAA Privacy Rule, 2003.

[15] L. Wang, Z. Cao, T. Okamoto, Y. Miao, and E. Okamoto. AuthorizationLimited Transformation-Free Proxy Cryptosystems and Their Security Analyses*. IEICE Transactions on Fundamentals of Electronics, Communications and Computer Sciences, (1):106-114, 2006.

[16] L. Zhou, M. A. Marsh, F. B. Schneider, and A. Redz. Distributed blinding for distributed elgamal re-encryption. In ICDCS '05: Proceedings of the 25th 
IEEE International Conference on Distributed Computing Systems, pages 824824. IEEE Computer Society, 2005. 


\section{Appendix A: An observation on the Matsuo scheme}

The Matsuo proxy re-encryption scheme assumes the delegator and deletee use the Boneh-Boyen Hierarchical IBE scheme [3] where the identity dimension is set to be 1 . The algorithms are as follows:

- Setup, which takes the security parameter $\ell$ as input, and outputs the public parameter $\left(g, g_{1}, g_{2}, h, p, \mathbb{G}, \mathbb{G}_{1}, \hat{e}\right)$ and the master key $m k$. Here, $\left(g, p, \mathbb{G}, \mathbb{G}_{1}, \hat{e}\right)$ is the basic bilinear map parameter, $g_{2}$ and $h$ are randomly chosen from $\mathbb{G}$, $m k=\alpha$ and $g_{1}=g^{\alpha}$ where $\alpha$ is randomly chosen from $\mathbb{Z}_{p}^{*}$. Note that the public parameter is an implicit input to all other algorithms, and we omit it in the description for simplicity.

- Keygen, which takes $m k$ and an identifier $I D_{t} \in \mathbb{Z}_{p}^{*}$ as input, and outputs the corresponding private key $s k_{t}$, where $\beta_{t}$ is randomly chosen from $\mathbb{Z}_{p}^{*}$ and

$$
\begin{aligned}
s k_{t} & =\left(g_{2}^{m k} \cdot\left(g_{1}^{I D_{t}} h\right)^{\beta_{t}}, g^{\beta_{t}}\right) \\
& =\left(g_{2}^{\alpha}\left(g_{1}^{I D_{t}} h\right)^{\beta_{t}}, g^{\beta_{t}}\right) \\
& =\left(d_{t 0}, d_{t 1}\right) .
\end{aligned}
$$

Note that $t$ is an integer used to index users in the system.

- Enc, which takes a message $m \in \mathbb{G}_{1}$ and an identifier $I D_{t} \in \mathbb{Z}_{p}^{*}$ as input, and outputs a ciphertext $c_{t}$, where $r$ is randomly chosen from $\mathbb{Z}_{p}^{*}$ and

$$
\begin{aligned}
c_{t} & =\left(g^{r},\left(g_{1}^{I D_{t}} h\right)^{r}, m \hat{e}\left(g_{1}, g_{2}\right)^{r}\right) \\
& =\left(c_{t 1}, c_{t 2}, c_{t 3}\right) .
\end{aligned}
$$

- Dec, which takes a ciphertext $c_{t}$ and the private key $s k_{t}$ as input, and outputs a message $m$ by computing

$$
m=\frac{c_{t 3} \hat{e}\left(d_{t 1}, c_{t 2}\right)}{\hat{e}\left(d_{t 0}, c_{t 1}\right)}
$$

Suppose Alice and Bob register under the same KGC, and possess identifier/private key pair $\left(I D_{i}, s k_{i}\right)$ and $\left(I D_{j}, s k_{j}\right)$, respectively. If Alice wants to delegate her decryption right to Bob, the algorithms of the Matsuo proxy re-encryption scheme [11] are as follows.

- Pkeygen, which takes $\left(m k, d_{j 1}\right)$ as input, and outputs the delegation key $r k_{i j}=$ $d_{j 1}^{\alpha}$.

- Preenc, which takes $r k_{i j}$ and $c_{i}$ as input, and outputs a new ciphertext $c_{j}$, where 


$$
\begin{aligned}
c_{j} & =\left(c_{i 1}, c_{i 2}, c_{i 3} \hat{e}\left(c_{i 1}^{I D_{j}-I D_{i}}, r k_{i j}\right)\right) \\
& =\left(c_{j 1}, c_{j 2}, c_{j 3}\right) .
\end{aligned}
$$

Given a re-encrypted ciphertext $c_{j}$, Bob can obtain the plaintext $m$ by running the IBE decryption algorithm.

$$
\begin{aligned}
m^{\prime} & =\frac{c_{j 3} \hat{e}\left(d_{j 1}, c_{j 2}\right)}{\hat{e}\left(d_{j 0}, c_{j 1}\right)} \\
& =\frac{m \hat{e}\left(g_{1}, g_{2}\right)^{r} \hat{e}\left(g^{r\left(I D_{j}-I D_{i}\right)}, g^{\alpha \beta_{j}}\right) \hat{e}\left(g^{\beta_{j}},\left(g_{1}^{I D_{i}} h\right)^{r}\right)}{\hat{e}\left(g_{2}^{\alpha}\left(g_{1}^{I D_{j}} h\right)^{\beta_{j}}, g^{r}\right)} \\
& =\frac{m \hat{e}\left(g^{\alpha r}, g_{2}\right) \hat{e}\left(g^{\alpha r\left(I D_{j}-I D_{i}\right)}, g^{\beta_{j}}\right) \hat{e}\left(g^{\beta_{j}}, g^{\alpha r I D_{i}} h^{r}\right)}{\hat{e}\left(g_{2}^{\alpha}\left(g^{\alpha I D_{j}} h\right)^{\beta_{j}}, g^{r}\right)} \\
& =\frac{m \hat{e}\left(g^{\alpha r}, g_{2}\right) \hat{e}\left(g^{\alpha r I D_{j}}, g^{\beta_{j}}\right) \hat{e}\left(g^{\beta_{j}}, h^{r}\right)}{\hat{e}\left(g_{2}^{r}, g^{\alpha}\right) \hat{e}\left(\left(g^{\alpha I D_{j}} h\right)^{\beta_{j}}, g^{r}\right)} \\
& =m .
\end{aligned}
$$

In the definition of Pkeygen, Alice is not required to be involved in generation of $r k_{i j}$, while the master key and Bob's private key is required in the generation of $r k_{i j}$. It is easy to verify that, the delegation key $r k_{i j}$ is capable of transforming any ciphertext, intended for any user registered at the KGC, into a decrytable ciphertext for Bob. As a result, once Alice has delegated her decryption right to Bob, then implicitly all users in the system have delegated their decryption right to Bob at the same time. 